\title{
Geometric Properties of a Class of Analytic Functions Defined by a Differential Inequality
}

\author{
Manpreet Kaur, Sushma Gupta, and Sukhjit Singh \\ Department of Mathematics, Sant Longowal Institute of Engineering and Technology, Longowal, Punjab 148106, India \\ Correspondence should be addressed to Manpreet Kaur; m.ahluwalia10@gmail.com
}

Received 8 July 2015; Revised 10 September 2015; Accepted 15 September 2015

Academic Editor: Baruch Cahlon

Copyright (C) 2015 Manpreet Kaur et al. This is an open access article distributed under the Creative Commons Attribution License, which permits unrestricted use, distribution, and reproduction in any medium, provided the original work is properly cited.

Let $\mathscr{A}$ be the class of analytic functions $f$ defined in the open unit disk $E$ and normalized by $f(0)=f^{\prime}(0)-1=0$. For $f(z) / z \neq 0$ in $E$, let $\mathscr{M}(\alpha, \lambda):=\left\{f \in \mathscr{A}:\left|-\alpha z^{2}(z / f(z))^{\prime \prime}+f^{\prime}(z)(z / f(z))^{2}-1\right| \leq \lambda, z \in E\right\}$, where $\lambda>0$ and $\alpha \in \mathbb{R} \backslash[-1 / 2,0]$. In the present paper, we find conditions under which functions in the class $\mathscr{M}(\alpha, \lambda)$ are starlike of order $\gamma, 0 \leq \gamma<1$.

\section{Introduction}

Let $\mathscr{A}$ denote the class of all functions $f$ that are analytic in the open unit disk $E=\{z:|z|<1\}$ and are normalized by the conditions $f(0)=f^{\prime}(0)-1=0$. We denote by $\delta$ the subclass of $\mathscr{A}$ consisting of functions which are univalent in $E$ and denote by $\mathcal{S}^{*}(\gamma), 0 \leq \gamma<1$, the class of functions in $\mathcal{S}$ which are starlike of order $\gamma$ in E. Analytically,

$$
\mathcal{S}^{*}(\gamma)=\left\{f \in \mathscr{A}: \operatorname{Re}\left(\frac{z f^{\prime}(z)}{f(z)}\right)>\gamma, z \in E\right\} .
$$

Note that $\delta^{*}(0)$ is the usual class of starlike (with respect to the origin) functions in $E$ and we denote it simply by $\mathcal{S}^{*}$. We say that $f \in R(\beta), 0<\beta \leq 1$, if $f \in \mathscr{A}$ and $\left|\arg f^{\prime}(z)\right|<\beta \pi / 2$, for all $z \in E$. It is well known that the functions in $R(1)$ are close-to-convex and hence univalent in $E[1,2]$.

In 1972, Ozaki and Nunokawa [3] studied the class $\mathscr{U}(\lambda)$, which is defined as

$$
\begin{aligned}
\mathscr{U}(\lambda) & \\
& =\left\{f \in \mathscr{A}:\left|f^{\prime}(z)\left(\frac{z}{f(z)}\right)^{2}-1\right| \leq \lambda, z \in E\right\},
\end{aligned}
$$

where $f(z) / z \neq 0$ in $E$. They proved that $\mathscr{U}(\lambda) \subset \delta$ for $0 \leq$ $\lambda \leq 1$. Several researchers studied this class (e.g., see [4-8]) and obtained many significant results.
Recently, Obradovic and Ponnusamy [9] investigated another class

$$
\begin{aligned}
& \mathscr{M}(\lambda)=\{f \\
& \in \mathscr{A}:\left|z^{2}\left(\frac{z}{f(z)}\right)^{\prime \prime}+f^{\prime}(z)\left(\frac{z}{f(z)}\right)^{2}-1\right| \leq \lambda, z \\
& \in E\},
\end{aligned}
$$

where $\lambda>0$ and $f(z) / z \neq 0$ in $E$. They obtained certain inclusion relations, characterization formula, and coefficient conditions. They also posed a question about the starlikeness of the functions in the class $\mathscr{M}(\lambda)$. The purpose of the present paper is to answer this question. In fact, we study a more general class

$$
\begin{aligned}
& \mathscr{M}(\alpha, \lambda)=\{f \\
& \in \mathscr{A}:\left|-\alpha z^{2}\left(\frac{z}{f(z)}\right)^{\prime \prime}+f^{\prime}(z)\left(\frac{z}{f(z)}\right)^{2}-1\right| \\
& \leq \lambda, z \in E\},
\end{aligned}
$$


where $f(z) / z \neq 0$ in $E, \lambda>0$, and $\alpha \in \mathbb{R} \backslash[-1 / 2,0]$. We find conditions on $\alpha, \lambda$, and $f$ involved in the class $\mathscr{M}(\alpha, \lambda)$ under which members of $\mathscr{M}(\alpha, \lambda)$ are starlike of a given order $\gamma, 0 \leq$ $\gamma<1$. We remark that the class $\mathscr{M}(\alpha, \lambda)$ follows essentially from the class $S(\alpha, \beta, \lambda)$ studied by Baricz and Ponnusamy [10] by taking $\beta=0$; however, we will study those issues for the class $\mathscr{M}(\alpha, \lambda)$ which are not studied by the authors for $S(\alpha, \beta, \lambda)$.

\section{Main Results}

Let $\mathscr{A}_{n}$ denote the class of analytic functions $p$ in $E$ such that $p^{(k)}(0)=0$ for $k=0,1,2, \ldots, n$, where $p^{(0)}(0)=p(0)$. With $w^{(0)}(0)=w(0)$, we set $\mathscr{B}_{n}=\{w: w$ is analytic, $|w(z)| \leq 1$ in $E$ and $w^{(k)}(0)=0$ for $\left.k=0,1,2, \ldots, n\right\}$. Functions in $\mathscr{B}_{n}$ are called Schwarz functions. Obviously, $w \in \mathscr{B}_{k}$ implies that $|w(z)| \leq|z|^{k+1}$ in $E$, for $k=0,1,2,3, \ldots, n$.

We begin with the following result.

Lemma 1. Let $f(z)=z+a_{2} z^{2}+a_{3} z^{3}+\cdots$ be in $M(\alpha, \lambda)$. Then, $\operatorname{Re}[f(z) / z] \geq 1 / 2$ in $E$ whenever $\left|a_{2}\right| \leq 1-\lambda /|2 \alpha+1|$.

Proof. As $f \in \mathscr{M}(\alpha, \lambda)$, there exists a Schwarz function $w \in$ $\mathscr{B}_{1}$ such that

$$
-\alpha z^{2}\left(\frac{z}{f(z)}\right)^{\prime \prime}+f^{\prime}(z)\left(\frac{z}{f(z)}\right)^{2}-1=\lambda w(z) .
$$

Since $w \in \mathscr{B}_{1}, w(0)=w^{\prime}(0)=0$. If we set

$$
\begin{aligned}
p(z) & =f^{\prime}(z)\left(\frac{z}{f(z)}\right)^{2}-1 \\
& =\left(\frac{z}{f(z)}\right)-z\left(\frac{z}{f(z)}\right)^{\prime}-1,
\end{aligned}
$$

where $p$ is an analytic function in $E$ with $p(0)=p^{\prime}(0)=0$, then (5) is equivalent to

$$
\alpha z p^{\prime}(z)+p(z)=\lambda w(z),
$$

from which we get

$$
p(z)=\frac{\lambda}{\alpha} \int_{0}^{1} w(t z) t^{1 / \alpha-1} d t
$$

or, equivalently,

$$
\begin{aligned}
& -z\left(\frac{z}{f(z)}-1+a_{2} z\right)^{\prime}+\left(\frac{z}{f(z)}-1+a_{2} z\right) \\
& =\frac{\lambda}{\alpha} \int_{0}^{1} w(t z) t^{1 / \alpha-1} d t .
\end{aligned}
$$

Solving this equation for $z / f(z)$, we obtain

$$
\frac{z}{f(z)}=1-a_{2} z-\frac{\lambda}{\alpha} \int_{0}^{1} \int_{0}^{1} \frac{w(s t z)}{s^{2}} t^{1 / \alpha-1} d s d t .
$$

Now, using the fact that $|w(s t z)| \leq|(s t z)|^{2}$, it follows that

$$
\begin{aligned}
\left|\frac{z}{f(z)}-1\right| & \leq\left|a_{2}\right||z|+\frac{\lambda}{|\alpha|} \int_{0}^{1} \int_{0}^{1} t^{1 / \alpha+1}|z|^{2} d s d t \\
& \leq|z|\left(\left|a_{2}\right|+\frac{\lambda|z|}{|2 \alpha+1|}\right), \quad z \in E .
\end{aligned}
$$

The inequality (11) is equivalent to

$$
\begin{gathered}
\left|\frac{f(z)}{z}-\frac{1}{1-|z|^{2}\left(\left|a_{2}\right|+\lambda|z| /|2 \alpha+1|\right)^{2}}\right| \\
\leq \frac{|z|\left(\left|a_{2}\right|+\lambda|z| /|2 \alpha+1|\right)}{1-|z|^{2}\left(\left|a_{2}\right|+\lambda|z| /|2 \alpha+1|\right)^{2}},
\end{gathered}
$$

which gives

$$
\begin{aligned}
\operatorname{Re}\left(\frac{f(z)}{z}\right) & \geq \frac{1}{1+|z|\left(\left|a_{2}\right|+\lambda|z| /|2 \alpha+1|\right)} \\
& >\frac{1}{1+\left(\left|a_{2}\right|+\lambda /|2 \alpha+1|\right)} \geq \frac{1}{2}
\end{aligned}
$$

for all $z \in E$ whenever $\left|a_{2}\right| \leq 1-\lambda /|2 \alpha+1|$.

In the next result, we find the range of values of $\lambda$ for which $f \in \mathscr{M}(\alpha, \lambda)$ implies that $f \in R(\beta), 0<\beta \leq 1$.

Theorem 2. Let $f(z)=z+a_{2} z^{2}+a_{3} z^{3}+\cdots$ be in $\mathscr{M}(\alpha, \lambda)$. Then, $f \in R(\beta)$ for $0<\lambda \leq \lambda_{\beta}$, where $\lambda_{\beta}$ satisfies the inequality

$$
\begin{aligned}
\sqrt{1-\frac{\lambda^{2}}{|2 \alpha+1|^{2}}} \sin \frac{\beta \pi}{2} \\
\quad-2\left(\left|a_{2}\right|+\frac{\lambda}{|2 \alpha+1|}\right) \sqrt{1-\left(\left|a_{2}\right|+\frac{\lambda}{|2 \alpha+1|}\right)^{2}} \\
\geq \frac{\lambda}{|2 \alpha+1|} \cos \frac{\beta \pi}{2} .
\end{aligned}
$$

Proof. From (8), we get

$$
|p(z)|<\frac{\lambda}{|2 \alpha+1|}, \quad z \in E,
$$

where

$$
p(z)=f^{\prime}(z)\left(\frac{z}{f(z)}\right)^{2}-1
$$

Therefore,

$$
\left|\arg f^{\prime}(z)\left(\frac{z}{f(z)}\right)^{2}\right| \leq \arcsin \left(\frac{\lambda}{|2 \alpha+1|}\right), \quad z \in E .
$$


Also, in view of (11), we obtain

$$
\left|\arg \left(\frac{z}{f(z)}\right)\right| \leq \arcsin \left(\left|a_{2}\right|+\frac{\lambda}{|2 \alpha+1|}\right), \quad z \in E .
$$

Therefore,

$$
\begin{aligned}
& \left|\arg f^{\prime}(z)\right| \leq\left|\arg f^{\prime}(z)\left(\frac{z}{f(z)}\right)^{2}\right|+2\left|\arg \left(\frac{z}{f(z)}\right)\right| \\
& \quad \leq \arcsin \left(\frac{\lambda}{|2 \alpha+1|}\right)+2 \arcsin \left(\left|a_{2}\right|+\frac{\lambda}{|2 \alpha+1|}\right) \\
& \quad=\arcsin \left(\frac{\lambda}{|2 \alpha+1|}\right)+\arcsin \left(2\left(\left|a_{2}\right|+\frac{\lambda}{|2 \alpha+1|}\right)\right. \\
& \left.\cdot \sqrt{1-\left(\left|a_{2}\right|+\frac{\lambda}{|2 \alpha+1|}\right)^{2}}\right) .
\end{aligned}
$$

Now, the desired result follows, if

$$
\begin{gathered}
\arcsin \left(\frac{\lambda}{|2 \alpha+1|}\right)+\arcsin \left(2\left(\left|a_{2}\right|+\frac{\lambda}{|2 \alpha+1|}\right)\right. \\
\cdot \sqrt{1-\left(\left|a_{2}\right|+\frac{\lambda}{|2 \alpha+1|}\right)^{2}} \leq \frac{\beta \pi}{2} .
\end{gathered}
$$

By using $\arcsin (x)+\arcsin (y)=\arcsin \left(x \sqrt{1-y^{2}}+y \sqrt{1-x^{2}}\right)$, $x, y \in[-1,1]$, and $x^{2}+y^{2} \leq 1$ and carrying out some simplifications, we conclude that (20) is equivalent to (14).

If $a_{2}=0$ and $\beta=1$, then Theorem 2 gives the following.

Corollary 3. If $f(z)=z+a_{3} z^{3}+\cdots$ is in $\mathscr{M}(\alpha, \lambda)$, and then $\operatorname{Re} f^{\prime}(z)>0$ in $E$, whenever $0<\lambda \leq|2 \alpha+1| / 2$.

Setting $a_{2}=0, \beta=1$, and $\alpha=-1$ in Theorem 2, we obtain the following.

Corollary 4. If $f(z)=z+a_{3} z^{3}+\cdots$ is in $\mathscr{M}(-1, \lambda)$, and then $\operatorname{Re} f^{\prime}(z)>0$ in $E$, whenever $0<\lambda \leq 1 / 2$.

In the following theorem, we find conditions under which functions in the class $\mathscr{M}(\alpha, \lambda)$ belong to $\mathcal{S}^{*}(\gamma), 0 \leq \gamma<1$.

Theorem 5. Let $f(z)=z+a_{2} z^{2}+a_{3} z^{3}+\cdots$ be in $M(\alpha, \lambda)$ and let $f^{\prime \prime}(0)=0$. Then, for $0 \leq \gamma<1, f \in \mathcal{S}^{*}(\gamma)$ provided $0<\lambda \leq(1-\gamma)|2 \alpha+1| /(\sqrt{2}+\gamma)$.

Proof. As $a_{2}=f^{\prime \prime}(0) / 2=0$, from (8) and (10), we get

$$
\begin{aligned}
\frac{z f^{\prime}(z)}{f(z)} & =\frac{f^{\prime}(z)(z / f(z))^{2}}{z / f(z)} \\
& =\frac{1+(\lambda / \alpha) \int_{0}^{1} w(t z) t^{1 / \alpha-1} d t}{1-(\lambda / \alpha) \int_{0}^{1} \int_{0}^{1}\left(w(s t z) / s^{2}\right) t^{1 / \alpha-1} d s d t} .
\end{aligned}
$$

Now, $\operatorname{Re}\left(z f^{\prime}(z) / f(z)\right)>\gamma$ is equivalent to

$$
\frac{z f^{\prime}(z) / f(z)-\gamma}{1-\gamma} \neq i T, \quad T \in \mathbb{R} .
$$

Using (21) in (22), we get

$$
\begin{aligned}
& \frac{\lambda}{\alpha} \int_{0}^{1} w(t z) t^{1 / \alpha-1} d t \\
& \quad-\gamma(1-i T)\left(1-\frac{\lambda}{\alpha} \int_{0}^{1} \int_{0}^{1} \frac{w(s t z)}{s^{2}} t^{1 / \alpha-1} d s d t\right) \\
& \quad+\frac{\lambda}{\alpha} i T \int_{0}^{1} \int_{0}^{1} \frac{w(s t z)}{s^{2}} t^{1 / \alpha-1} d s d t \neq-(1-i T)
\end{aligned}
$$

or, equivalently,

$$
\begin{aligned}
& \frac{\lambda}{2 \alpha}\left[\int_{0}^{1} w(t z) t^{1 / \alpha-1} d t-\int_{0}^{1} \int_{0}^{1} \frac{w(s t z)}{s^{2}} t^{1 / \alpha-1} d s d t\right] \\
& -\gamma\left[1-\frac{\lambda}{\alpha} \int_{0}^{1} \int_{0}^{1} \frac{w(s t z)}{s^{2}} t^{1 / \alpha-1} d s d t\right]+\frac{\lambda}{2 \alpha} \\
& \quad \cdot \frac{1+i T}{1-i T}\left[\int_{0}^{1} w(t z) t^{1 / \alpha-1} d t\right. \\
& \left.+\int_{0}^{1} \int_{0}^{1} \frac{w(s t z)}{s^{2}} t^{1 / \alpha-1} d s d t\right] \neq-1 .
\end{aligned}
$$

If we denote the left-hand side of (24) by $H(w, T, z)$ and let

$$
M=\sup _{T \in \mathbb{R}, w \in \mathscr{B}_{1}, z \in E}|H(w, T, z)|,
$$

then, in view of the rotation invariance property of the set $\mathscr{B}_{1}$, we obtain that (22) holds if $M \leq 1$.

A simple calculation gives

$$
\begin{aligned}
M & \leq \sup _{w \in \mathscr{B}_{1}, z \in E} \frac{\lambda}{2|\alpha|} \mid \int_{0}^{1} w(t z) t^{1 / \alpha-1} d t \\
& -\int_{0}^{1} \int_{0}^{1} \frac{w(s t z)}{s^{2}} t^{1 / \alpha-1} d s d t \mid \\
& +\frac{\lambda}{2|\alpha|} \mid \int_{0}^{1} w(t z) t^{1 / \alpha-1} d t \\
& +\int_{0}^{1} \int_{0}^{1} \frac{w(s t z)}{s^{2}} t^{1 / \alpha-1} d s d t|+\gamma| 1-\frac{\lambda}{\alpha} \\
& \cdot \int_{0}^{1} \int_{0}^{1} \frac{w(s t z)}{s^{2}} t^{1 / \alpha-1} d s d t \mid .
\end{aligned}
$$

Now, by the parallelogram Law, $\left|z_{1}+z_{2}\right|+\left|z_{1}-z_{2}\right| \leq$ $2 \sqrt{\left|z_{1}\right|^{2}+\left|z_{2}\right|^{2}}$, we have 


$$
M \leq \frac{\lambda}{|\alpha|} \sup _{w \in \mathscr{B}_{1}, z \in E}\left[\sqrt{\left|\int_{0}^{1} w(t z) t^{1 / \alpha-1} d t\right|^{2}+\left|\int_{0}^{1} \int_{0}^{1} \frac{w(s t z)}{s^{2}} t^{1 / \alpha-1} d s d t\right|^{2}}\right]+\gamma+\frac{\lambda \gamma}{|\alpha|}\left|\int_{0}^{1} \int_{0}^{1} \frac{w(s t z)}{s^{2}} t^{1 / \alpha-1} d s d t\right|
$$

Using the fact that $|w(z)| \leq|z|^{2}$ in $E$, we get

$$
\begin{aligned}
M & \leq \frac{\lambda}{|\alpha|} \\
& \cdot \sup _{z \in E} \sqrt{\left(\int_{0}^{1}|z|^{2} t^{1 / \alpha+1} d t\right)^{2}+\left(\int_{0}^{1} \int_{0}^{1}|z|^{2} t^{1 / \alpha+1} d t\right)^{2}} \\
& +\gamma+\frac{\lambda \gamma}{|\alpha|} \int_{0}^{1} \int_{0}^{1}|z|^{2} t^{1 / \alpha+1} d t \\
= & \frac{\lambda(\sqrt{2}+\gamma)}{|2 \alpha+1|}+\gamma .
\end{aligned}
$$

Thus, $M \leq 1$, if $\lambda \leq(1-\gamma)|2 \alpha+1| /(\sqrt{2}+\gamma)$.

Taking $\gamma=0$, in Theorem 5, we get the following result.

Corollary 6. Let $f$ be as in Theorem 5. Then, $f \in S^{*}$ provided $0<\lambda \leq|2 \alpha+1| / \sqrt{2}$.

\section{Conflict of Interests}

The authors declare that there is no conflict of interests regarding the publication of this paper.

\section{References}

[1] K. Noshiro, "On the theory of Schlicht functions," Journal of the Faculty of Science, Hokkaido University, vol. 2, no. 3, pp. 129-155, 1934-1935.

[2] S. E. Warchawski, "On the higher derivatives at the boundary in conformal mapping," Transactions of the American Mathematical Society, vol. 38, no. 2, pp. 310-340, 1935.

[3] S. Ozaki and M. Nunokawa, "The Schwarzian derivative and univalent functions," Proceedings of the American Mathematical Society, vol. 33, no. 2, pp. 392-394, 1972.

[4] M. Obradović, "A class of univalent functions," Hokkaido Mathematical Journal, vol. 27, no. 2, pp. 329-335, 1998.

[5] M. Obradovic, S. Ponnusamy, V. Singh, and P. Vasundhra, "Univalency, starlikeness and convexity applied to certain classes of rational functions," Analysis: International Mathematical Journal of Analysis and its Applications, vol. 22, no. 3, pp. 225-242, 2002.

[6] S. Ponnusamy and P. Vasundhra, "Univalent functions with missing Taylor coefficients," Hokkaido Mathematical Journal, vol. 33, no. 2, pp. 341-355, 2004.

[7] S. Ponnusamy and P. Vasundhra, "Criteria for univalence, starlikeness and convexity," Annales Polonici Mathematici, vol. 85, no. 2, pp. 121-133, 2005.

[8] V. Singh, "On a class of univalent functions," International Journal of Mathematics and Mathematical Sciences, vol. 23, no. 12, pp. 855-857, 2000.
[9] M. Obradovic and S. Ponnusamy, "A class of univalent functions defined by a differential inequality," Kodai Mathematical Journal, vol. 34, no. 2, pp. 169-178, 2011.

[10] Á. Baricz and S. Ponnusamy, "Differential inequalities and Bessel functions," Journal of Mathematical Analysis and Applications, vol. 400, no. 2, pp. 558-567, 2013. 


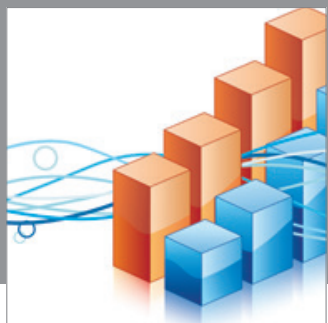

Advances in

Operations Research

mansans

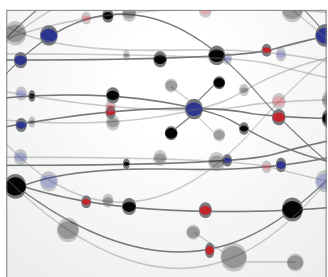

The Scientific World Journal
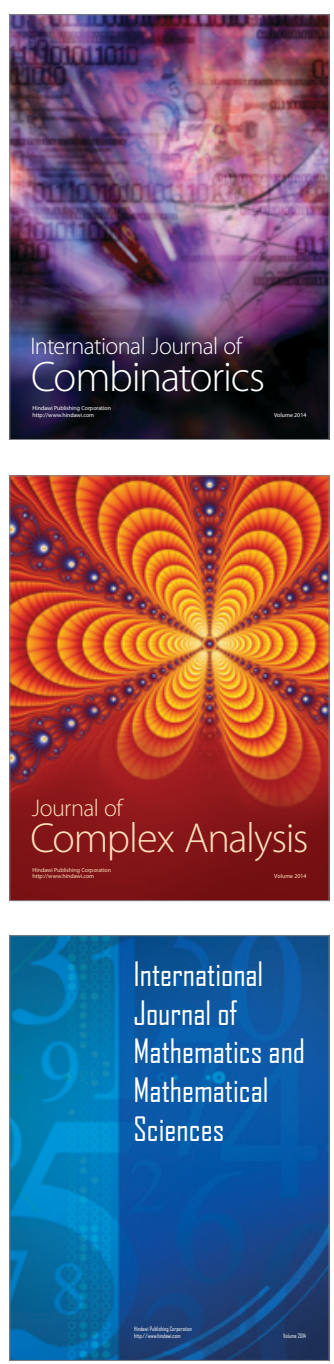
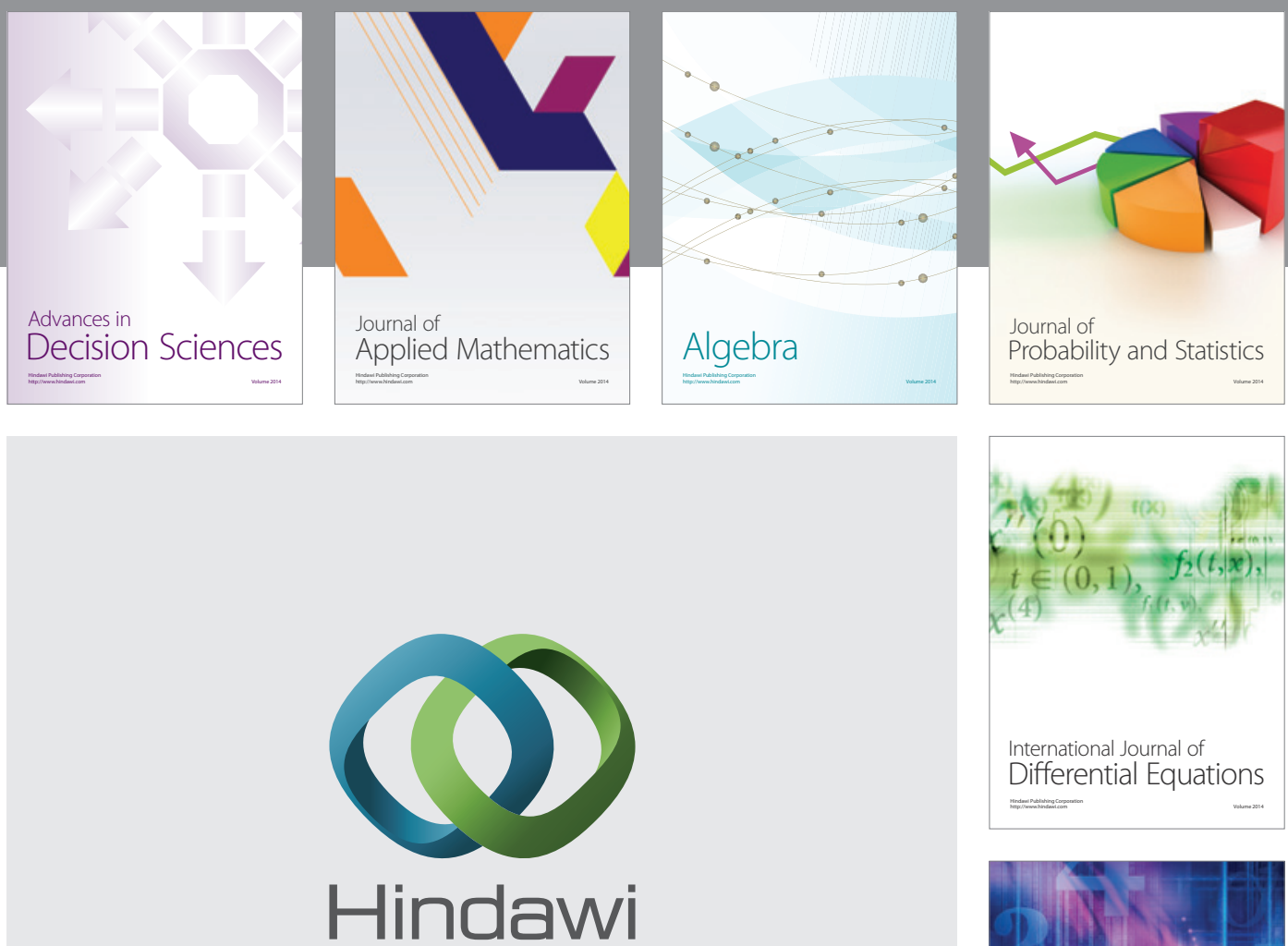

Submit your manuscripts at http://www.hindawi.com
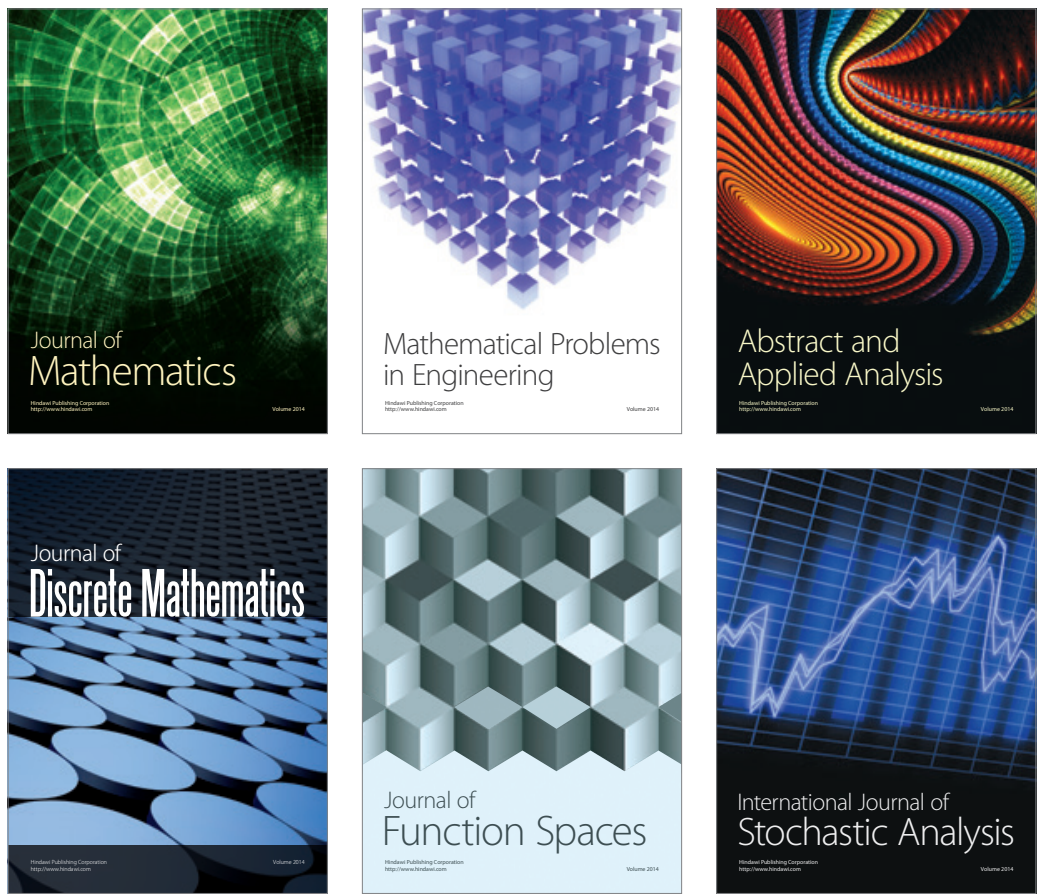

Journal of

Function Spaces

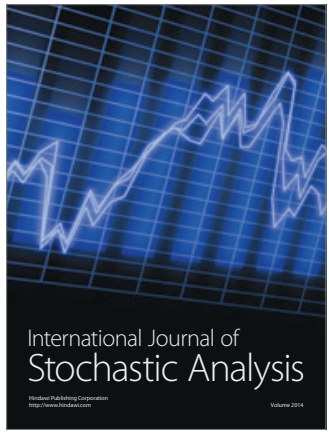

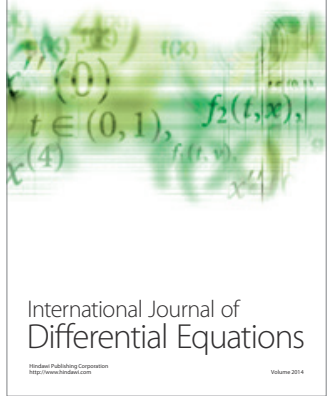
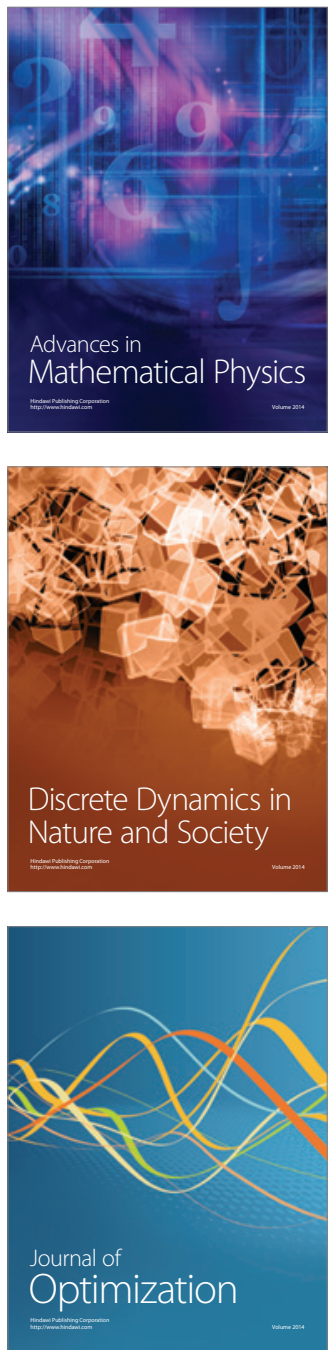